\title{
Item-Level Investigation of Participant and Study Partner Report on the Cognitive Function Index from the A4 Study Screening Data
}

\author{
R.E. Amariglio 1,2, S.A.M. Sikkes ${ }^{6}$, G.A. Marshall1,2, R.F. Buckley 2,7,8, J.R. Gatchel ${ }^{3,4}$, K.A. Johnson ${ }^{1,5}$, \\ D.M. Rentz ${ }^{1,2}$, M.C. Donohue ${ }^{9}$, R. Raman ${ }^{9}$, C.-K. Sun ${ }^{9}$, R. Yaari ${ }^{10}$, K.C. Holdridge ${ }^{10}$, J.R. Sims ${ }^{10}$, J.D. Grill ${ }^{11}$, \\ P.S. Aisen ${ }^{9}$, R.A. Sperling ${ }^{1,2}$ and the A4 Study Team

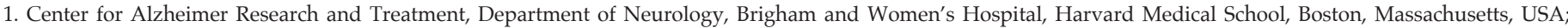

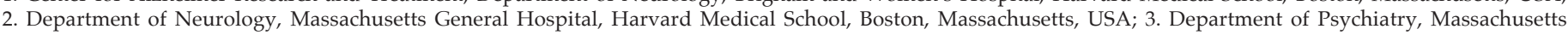

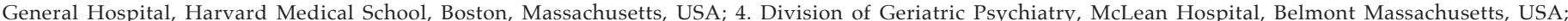

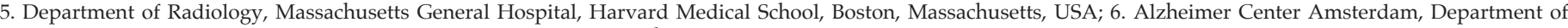

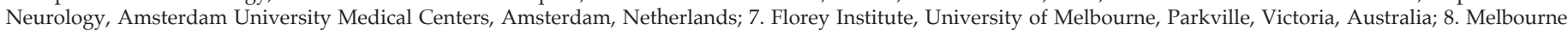

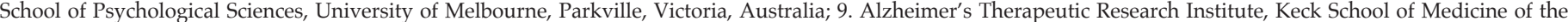 \\ University of Southern California, San Diego, CA, USA; 10. Eli Lilly and Company, Indianapolis, IN, USA; 11. University of California Irvine, Irvine, USA
}

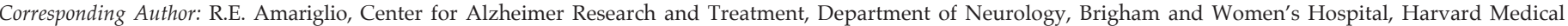
School, Boston, Massachusetts, 02115, USA, ramariglio@partners.org

\begin{abstract}
BACKGROUND: Greater subjective cognitive changes on the Cognitive Function Index (CFI) was previously found to be associated with elevated amyloid $(A B)$ status in participants screening for the A4 Study, reported by study partners and the participants themselves. While the total score on the CFI related to amyloid for both sources respectively, potential differences in the specific types of cognitive changes reported by either participants or their study partners was not investigated.

OBJECTIVES: To determine the specific types of subjective cognitive changes endorsed by participants and their study partners that are associated with amyloid status in individuals screening for an AD prevention trial.
\end{abstract}

DESIGN, SETTING, PARTICIPANTS: Four thousand four hundred and eighty-six cognitively unimpaired (CDR $=0$; MMSE 25-30) participants (ages 65-85) screening for the A4 Study completed florbetapir (AB) Positron Emission Tomography (PET) imaging. Participants were classified as elevated amyloid $(A ß+; n=1323)$ or non-elevated amyloid $(A ß-; n=3163)$.

MEASUREMENTS: Prior to amyloid PET imaging, subjective report of changes in cognitive functioning were measured using the CFI (15 item questionnaire; Yes/Maybe/No response options) and administered separately to both participants and their study partners (i.e., a family member or friend in regular contact with the participant). The impact of demographic factors on CFI report was investigated. For each item of the CFI, the relationship between $A ß$ and $C F I$ response was investigated using an ordinal mixed effects model for participant and study partner report.

RESULTS: Independent of $A ß$ status, participants were more likely to report 'Yes' or 'Maybe' compared to the study partners for nearly all CFI items. Older age $(\mathrm{r}=0.06, \mathrm{p}<0.001)$ and lower education $(\mathrm{r}=-0.08, \mathrm{p}<0.001)$ of the participant were associated with higher CFI. Highest coincident odds ratios related to $\mathrm{A} B+$ for both respondents included items assessing whether 'a substantial decline in memory' had occurred in the last year $(\mathrm{ORsp}=1.35$ [95\% CI 1.11, 1.63]; ORp $=1.55$ [95\% CI 1.34, 1.79] $)$ and whether the participant had 'seen a doctor about memory' $(\mathrm{ORsp}=1.56$ [95\% CI 1.25, 1.95]; ORp $=1.71$ [95\% CI 1.37, 2.12]). For two items, associations were significant for only study partner report; whether the participant 'Repeats questions'
$(\mathrm{ORsp}=1.30[95 \% \mathrm{CI} 1.07,1.57])$ and has 'trouble following the news' $(\mathrm{ORsp}=1.46[95 \%$ CI 1.12, 1.91]). One question was significant only for participant report; 'trouble driving' (ORp= 1.25 [95\% CI 1.04, 1.49]).

CONCLUSIONS: Elevated $\mathrm{A} \beta$ is associated with greater reporting of subjective cognitive changes as measured by the CFI in this cognitively unimpaired population. While participants were more likely than study partners to endorse change on most CFI items, unique CFI items were associated with elevated $A ß$ for participants and their study partners, supporting the value of both sources of information in clinical trials.

Key words: Subjective cognitive cecline, amyloid, clinical trial, Alzheimer's disease.

\section{Introduction}

A fter a series of disappointing results at the symptomatic stages of Alzheimer's disease (AD), therapeutic trials are increasingly moving towards prevention at the preclinical stage (1). Individuals enrolled in a secondary prevention trial are characterized as clinically normal, but are considered at increased risk of $\mathrm{AD}$ due to elevated biomarkers, such as amyloid (Aß) Positron Emission Tomography (PET) imaging. By shifting the focus earlier in the disease, however, demonstrating clinically meaningful treatment effects is challenging, since most individuals at the preclinical stage are not expected to demonstrate overt cognitive and functional impairment during the course of a trial (2). As such, efforts to identify new methods to capture subtle changes in cognitive functioning prior to the onset of objective clinical impairment are needed to quantify treatment effects with greater resolution.

Subjective report of everyday high-level cognitive functioning from both the individual and a close family member or friend, may offer a window into early 
cognitive changes along the preclinical stage. Indeed, prior studies have shown that both greater cognitive complaints from the participant, as well as from a study partner, are associated with higher likelihood of subsequent cognitive decline and clinical progression (3-6).

In addition to serving as outcome measures, subjective cognitive assessments may also facilitate the process of identifying individuals who meet criteria for preclinical AD. As individuals age, cognitive complaints become increasingly common and are not necessarily specific to a pathological process (7). Elucidating particular patterns of complaints from the participant and the study partner that relate to $\mathrm{AD}$ biomarkers may enhance the utility of subjective report that is sometimes dismissed for being non-specific $(8,9)$. Further, better characterization of the subtle changes that are observed at the preclinical stage may ultimately help to identify specific targets for therapeutic intervention.

In the current study, we examined data from individuals screening for the Anti-Amyloid Asymptomatic Alzheimer's (A4) Study testing solanezumab, an anti-amyloid antibody, in a secondary $\mathrm{AD}$ prevention trial. In particular, we sought to build upon previous findings in the A4 screen data that found both participant and study partner report related to $A B$ on the total score of the Cognitive Function Index (CFI) (10), a subjective questionnaire that asks a participant and study partner about change in the participant's cognitive functioning over the last year $(3,11)$. Here, we investigated the potential impact of demographic factors on participant vs. study partner report on the total score of the CFI, as well as which specific individual items on the CFI related to amyloid burden on PET. In this way, we aimed to elucidate the pattern of cognitive complaints at the preclinical stage of $\mathrm{AD}$ from both the perspective of the participant and study partner.

\section{Methods}

Data presented here come from participants who were screened for the A4 Study. In brief, the A4 Study is a preclinical stage treatment trial that is being conducted at 67 clinical trial sites in the U.S., Canada, Japan, and Australia, among participants with elevated $A B$ as determined by florbetapir PET. Participants first underwent an initial clinic screening visit and if eligibility criteria were met, subsequently underwent $A B$ PET imaging at a second screening visit. Participants who completed screening for the A4 study, were ages 65-85 years and were considered cognitively unimpaired, based on a global CDR (12) score of 0, Mini-Mental State Exam (MMSE) (13) score of 25-30, and Logical Memory II subscale delayed paragraph recall (LM-IIa) of the Wechsler Memory Scale-Revised (WMS-R) (14) score of 6-18. Moreover, participants did not have unstable or exclusionary medical or psychiatric problems.
Participants had adequate literacy in English, Spanish, or Japanese, and had adequate vision and hearing to complete the required cognitive tests. Participants were required to have a study partner who was willing to provide collateral information about the participant's everyday cognitive functioning; study partners were required to have at least weekly contact with participants in person, by phone, or by email. Key exclusion criteria for participants were diagnosis of cognitive impairment or dementia, use of AD medications, unstable anxiety or depression, or other unstable medical conditions, although participants with treated hypertension, diabetes, and other common medical ailments were permitted. Four thousand four hundred and eighty-six participants meeting these criteria then underwent florbetapir PET imaging.

\section{Cognitive Function Index}

The CFI was originally developed as a 14-item, selfadministered mail-in screening instrument for AD diagnostic evaluation in prevention trials (10). The CFI has a participant version in which participants report on their own cognitive functioning, as well as a study partner version in which study partners report on the participants' cognitive functioning. The CFI was previously found to have adequate validity and reliability $(6,10)$. All questions on the CFI ask about cognitive changes over the last year with response options that include Yes (2), No (0), and Maybe (1). Questions range from cognitive items (e.g., "Compared to one year ago, do you feel that your memory has declined substantially?") to functional items (e.g., "Compared to one year ago, do you have more difficulty managing money?"). The A4 version of the CFI added an additional question, "In the past year, have you seen a doctor about memory concerns?" with response options: Yes (1) or No (0). On a few questions, (e.g., "Has your work performance (paid or volunteer) declined significantly, compared to one year ago?"), Non-Applicable (N/A) was also a response option. A total CFI score can be derived by summing each item of the participant and study partner versions of the questionnaire respectively (the range is 0-29 with higher scores indicating greater complaints about cognitive functioning difficulties). The CFI was administered separately to both the participant and their study partner at the first screen visit prior to florbetapir PET imaging at the second screening visit.

\section{Amyloid PET Imaging}

Florbetapir PET was acquired 50-70 minutes after injection of $10 \mathrm{mCi}$ of florbetapir F 18. Amyloid eligibility (elevated $[\mathrm{A} \beta+]$ and eligible to continue in screening vs. not elevated [AB-] and ineligible) was assessed using an algorithm combining both quantitative standardized 
Table 1. Demographic variables of all participants with comparison of Not elevated (Aß-) and elevated amyloid (Aß+) groups

\begin{tabular}{|c|c|c|c|c|}
\hline Table 1 - Demographics & $\begin{array}{l}\text { All Amyloid PET participants } \\
\qquad \mathrm{N}=4486\end{array}$ & $\begin{array}{l}\text { Not Elevated Amyloid (Aß-) } \\
\qquad \mathrm{N}=3163\end{array}$ & $\begin{array}{l}\text { Elevated Amyloid(Aß+) } \\
\qquad N=1323\end{array}$ & P-value $A ß$ - vs $A ß+$ \\
\hline Age - Mean years (S.D.) & $71.29(4.67)$ & $70.95(4.53)$ & $72.10(4.89)$ & $<0.0001$ \\
\hline Education - Mean years (S.D.) & $16.58(2.84)$ & $16.60(2.85)$ & $16.54(2.81)$ & 0.5327 \\
\hline PET SUVr Mean (S.D.) & $1.09(0.19)$ & $0.99(0.07)$ & $1.33(0.18)$ & $<0.000^{1}$ \\
\hline Sex & & & & $0.641^{1}$ \\
\hline $\mathrm{F}$ & $2663(59 \%)$ & $1885(60 \%)$ & $778(59 \%)$ & \\
\hline White & $4116(92 \%)$ & $2866(91 \%)$ & $1250(94 \%)$ & $<0.001^{1}$ \\
\hline Asian & $171(4 \%)$ & $141(4 \%)$ & $30(2 \%)$ & $<0.001^{1}$ \\
\hline Black or African American & $167(4 \%)$ & $141(4 \%)$ & $30(2 \%)$ & $0.007^{1}$ \\
\hline American Indian or Alaskan Native & $32(1 \%)$ & $22(1 \%)$ & $32(1 \%)$ & $0.846^{1}$ \\
\hline Native Hawaiian & $2(0 \%)$ & $0(0 \%)$ & $2(0 \%)$ & $1.00^{1}$ \\
\hline Marital Status & & & & $0.655^{1}$ \\
\hline Married & $3166(71 \%)$ & $2223(70 \%)$ & $943(71 \%)$ & \\
\hline SP relationship & & & & 0.543 \\
\hline Spouse & $2767(62 \%)$ & $1948(62 \%)$ & $819(62 \%)$ & \\
\hline Adult child & $526(12 \%)$ & $236(13 \%)$ & $360(11 \%)$ & \\
\hline Friend / companion & $849(19 \%)$ & $613(19 \%)$ & $236(18 \%)$ & \\
\hline \multicolumn{5}{|l|}{ Living Situation of SP } \\
\hline Living with Participant & $3019(67 \%)$ & $2131(67 \%)$ & $888(67 \%)$ & 0.889 \\
\hline \multicolumn{5}{|l|}{ SP Gender } \\
\hline
\end{tabular}

1. Fisher's Exact test

uptake value ratio (SUVr) methodology and qualitative visual read performed at a central laboratory. Mean cortical standardized uptake value ratio [SUVr] using a whole cerebellar reference region of $\geq 1.15$ was utilized to define elevated amyloid as the primary criterion, as quantitative assessment was thought to be more sensitive to the presence of early amyloidosis in the preclinical stage of AD. A SUVr between 1.10 and 1.15 was considered to be elevated amyloid only if the visual read was considered positive by a two independent-reader consensus determination (10).

\section{Statistical Analyses}

Demographic factors of the participant (e.g., sex, age, education) and of the study partner (e.g., partner sex, partner age, living status with participant) were summarized by $A ß$ status with means, standard deviations, and two-sample t-test; or counts, percentages, and Fisher's Exact test. Pearson's correlation coefficients were calculated for continuous characteristics and CFI scores. Linear regression models were used to determine whether there was an interaction between $A ß$ status and each participant and study partner characteristic on the CFI score for the participant and study partner report.

To compare level of endorsement on each item of the CFI between participant and study partner, a cumulative odds mixed effects model was employed with CFI response $(\mathrm{No}=0, \mathrm{Maybe}=1, \mathrm{Yes}=2)$ as the outcome and CFI source (participant or study partner), age, sex, and education as the predictors; and dyad-specific random intercepts. Non-applicable data was treated as missing. Item level missing data was rare and was not analyzed with imputation. To determine the relationship between $A B$ status and level of endorsement on each item, separate cumulative odds models were fit for each CFI item. CFI response for participant and study partner were run separately as the dependent variable and $A B$ status as the predictor controlling for age, education, and sex. The false discovery rate for item level analysis was adjusted within source (participant vs. study partner) by the method of Benjamini and Hochberg (1995) (15). Statistical analyses were performed using the R software (http:/ / www.r-project.org). 


\section{Results}

Of the 4486 participants, 1322 were categorized as $A ß+(29.5 \%)$ and 3163 were $A ß-. A ß+$ participants were slightly older than Aß- (see table 1). No differences between $A B$ groups were observed by sex, years of education, or marital or retirement status. Study partners were majority spouses $(62 \%)$ and female $(60 \%)$ and were age $65.8 \pm 11.2$ years. There were no differences in relationship to study partner by Aß group.

Higher score on the participant CFI was associated with older age $(\mathrm{r}=0.06, \mathrm{p}<0.001)$ and lower education $(\mathrm{r}=-0.08, \mathrm{p}<0.001)$, but was not associated with sex $(\mathrm{t}=-$ $2.68, \mathrm{p}=0.79)$. Study partner CFI score was higher for female study partners (meanfemale $=2.94$, meanmale $=$ 2.54; $\mathrm{t}=3.5, \mathrm{p}=0.0005$ ) and if a study partner lived with the participant (meanlive with $=2.92$, meanlive separate $=$ $2.49 ; \mathrm{t}=-3.7, \mathrm{p}=0.0003)$. Older age of the participant related to higher study partner CFI score $(r=0.07$, $\mathrm{p}<0.001$ ).

Next, we were interested in whether demographic factors modified the relationship between $A B$ and the CFI total score for participant report and study partner report respectively. When examining participant report, there was not a significant interaction between $A B$ and sex $(\beta=-0.14, p=0.30), A \beta$ and age $(\beta=0.015, p=0.27)$, nor $A B$ and education level of participant $(B=0.106, p=$ $0.47)$ to predict participant CFI score. For study partners, there was not a significant interaction between $A B$ and sex of the study partner $(B=0.05, p=0.85)$ nor $A B$ and living situation of study partner with participant $(B=$ $0.17, p=0.53$ ). The interaction between $A B$ and age of study partner to predict study partner CFI score was at the significance nominal threshold $(B=0.02, p=0.05)$. Specifically, among $A ß-$-, younger study partners tended to report higher CFI scores. However, among $A ß+$, age of study partner did not modify CFI report.

At the item level, the 3 most commonly endorsed ('Yes' or 'Maybe' response) items were the same for both participant and study partner report (see figure : 'trouble with names and words' 'relying on written reminders' and 'misplacing things' (See figure 1, supplemental table 1). The 3 least endorsed items were also the same for participant and study partner report: 'managing money' 'difficulty with hobbies' 'difficulty with appliances'. For one item, 'trouble with work performance,' $18 \%$ of participants and $25 \%$ of study partners did not find this item applicable.

In general, greater endorsement was found for participants compared to their study partners (see figure 1). The only items that did not significantly differ in level of endorsement between participant and study partner included 'Seen a doctor about memory,' 'Repeating questions,' 'Difficulty with appliance and electronic devices.'
Figure 1. Percentage of endorsement on CFI for participant and study partner report at the item level

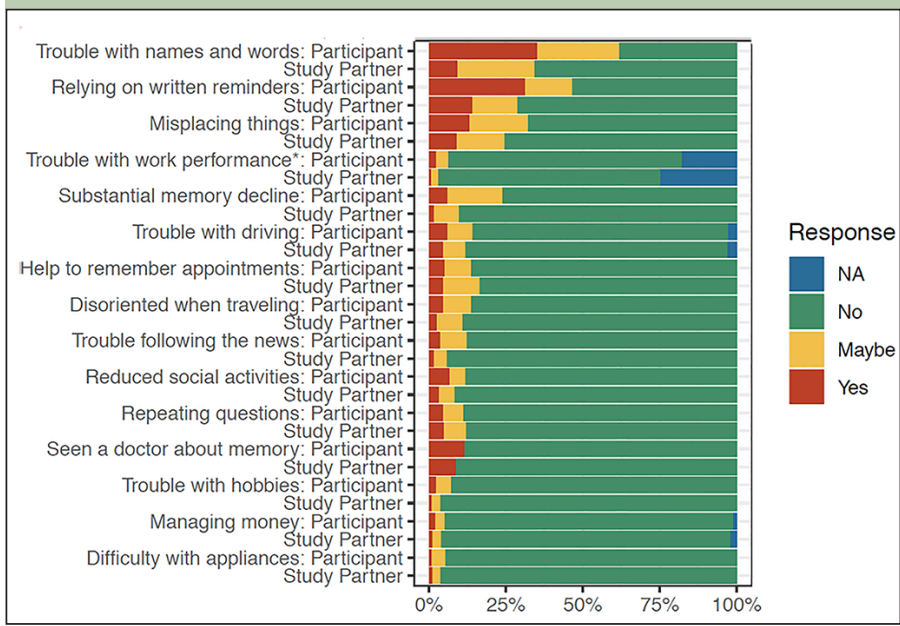

Figure 2. CFI items and odds of endorsement related to $A ß$

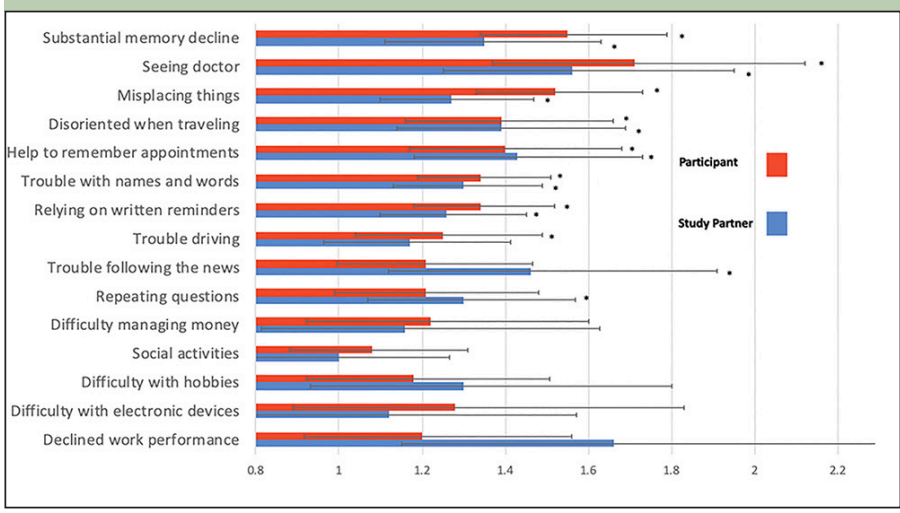

Items presented highest odds of endorsement and $\mathrm{AB}+$ to lowest for participant and study partner report. ${ }^{*}=$ significant association of item and $\mathrm{AB}+$

As was previously reported (10), $A ß+$ participants reported higher total CFI (Cohen $\mathrm{d}=0.31, \mathrm{p}<0.001$ ) and study partners reported $A ß+$ participants had higher total CFI (Cohen $d=0.23, p<0.001$ ). Here, we examined the relationship between $A B$ status and level of endorsement for each item. For 7 of the 15 items, $A ß+$ was associated with a higher odds of endorsement on CFI for both participant and study partner report and included: 'Seen a doctor about memory,' 'Substantial memory decline,' 'Misplacing things,' 'Help to remember appointments,' 'Disoriented when traveling,' 'Trouble with names and words,' and 'Relying on written reminders.' For participant report, but not study partner, $A B+$ was associated with greater endorsement on 'trouble with driving.' For study partner report but not participant, $A ß+$ was associated with 'trouble following the news' and 'repeating questions' (see figure 2). Additionally, $\mathrm{A} ß+$ was associated with 'decline in work performance' but as noted above $25 \%$ of study partners answered 'not applicable' leading to less stable estimates on this item compared to other items on the CFI. All items 
significantly associated with $A ß+$ remained significant after an FDR adjustment.

\section{Discussion}

Extending previous findings from the A4 screen data that found for $\mathrm{Aß}+$ participants, participant and study partner CFI scores were higher than for Aß- participants (10), here we found at an item-level, 7 of the 15 CFI items were related to $A ß+$ for participant and study partner report. Further, the odds of endorsement associated with elevated $A B$ was numerically higher for participant report compared to study partner report. Items that were related to both study partner and participant report reflected predominantly cognitive changes (e.g., 'Substantial memory decline,' 'Misplacing things'). Functional items (e.g., 'Hobbies more difficult,' 'Reduced social activities,' 'Difficulty managing money,' and 'Difficulty with appliances') were much less likely to be endorsed by either a participant or a study partner and were not associated with $\mathrm{A} ß+$.

Additionally, the item 'seen a doctor about memory' related to $A ß+$ for both participant and study partner report. Unlike the other CFI items, this question is based on a specific event rather than an overall subjective experience. The association of 'seen a doctor about memory' with $A B$ is consistent with previous work demonstrating individuals recruited from a memory clinic are more likely $\mathrm{A} ß+$ compared to community-based individuals with subjective memory complaints $(9,16)$.

Several items were only related to study partner or participant report alone. Specifically, two items were related to $A ß+$ for study partner report (i.e., 'Repeating questions' and 'trouble following the news'). Interestingly, the fact that study partners report 'repeating questions,' but not participants themselves is consistent with clinical observations, in that the participant may not realize they are repeating themselves and would be less likely to endorse this item.

These findings suggest that, even at the preclinical phase, subtle changes in cognitive functioning are recognized by and at the level of awareness of some participants and some study partners. Participant report, while potentially sensitive at the earliest stages of disease, has faced criticism as an outcome in clinical trials as changes in self-awareness (i.e., anosognosia) in patient reported outcomes can occur, particularly by the stage of dementia (17). Thus, there have been concerns as to whether participant report can reliably serve as an indicator of symptom progression for the duration of a trial as individuals move towards clinical impairment. Conversely, it has been unclear what changes if any, a study partner can observe at the preclinical stage when individuals are entirely independent in their daily activities. Importantly, in this study of individuals screening for a prevention trial, study partner report was consistent with participant report, despite the fact that individuals had a global CDR score of 0 .

We also examined the impact of demographic features of participants and their study partners on the relationship between $A B$ status and CFI total score. For participants, while there was a higher level of endorsement for CFI that related to older age and lower education, these demographic variables did not significantly modify the relationship between Aß status and CFI score. Likewise, there was a higher level of endorsement for study partner-reported CFI if the study partner was female or lived with the participant, however these demographic variables did not significantly modify the relationship between $A ß$ status and CFI score. Finally, while age of the study partner did not relate to study partner reported CFI score, there was a nominally significant modifying effect of study partner age on the relationship between $A B$ status and CFI score. Taken together, the associations between $\mathrm{A} \beta+$ and CFI seem to remain even after after adjusting for age, education, sex, and living situation of study partner with participant.

A few limitations to this study a worth noting. Participants in this study were highly educated with limited ethnic and racial diversity, typical for clinical trial populations. Thus, it remains unknown how these findings might generalize to the larger population, as there may be educational and cultural differences in the value of subjective reporting as it relates to risk for AD.

Our findings are in support of subjective report of cognitive functioning to characterize early manifestations of $\mathrm{AD}$ among those in an early-treatment trial. While participant report appears to be numerically higher as it relates to $A B$ status compared study partner report, study partners are also observing similar changes as they relate to $A B$ status. In the future, examining items longitudinally with tau PET in combination with amyloid PET will also help to better approximate the optimal utility of participant and study partner report as individuals decline or improve during the course of a trial.

Funding: The A4 Study is a secondary prevention trial in preclinical Alzheimer's disease, aiming to slow cognitive decline associated with brain amyloid accumulation in clinically normal older individuals. The A4 Study is funded by a public-private-philanthropic partnership, including funding from the National Institutes of Health-National Institute on Aging (U19 AG010483, U24AG057437, R01 AG063689), Eli Lilly and Company, Alzheimer's Association, Accelerating Medicines Partnership, GHR Foundation, an anonymous foundation and additional private donors, with in-kind support from Avid and Cogstate. The companion observational Longitudinal Evaluation of Amyloid Risk and Neurodegeneration (LEARN) Study is funded by the Alzheimer's Association (LEARN-15-338729) and GHR Foundation. The A4 and LEARN Studies are led by Dr. Reisa Sperling at Brigham and Women's Hospital, Harvard Medical School and Dr. Paul Aisen at the Alzheimer's Therapeutic Research Institute (ATRI), University of Southern California. The A4 and LEARN Studies are coordinated by ATRI at the University of Southern California, and the data are made available through the Laboratory for Neuro Imaging at the University of Southern California. The participants screening for the A4 Study provided permission to share their de-identified data in order to advance the quest to find a successful treatment for Alzheimer's disease.

Acknowledgements: We would like to acknowledge the dedication of all the participants, the site personnel, and all of the partnership team members who continue to make the A4 and LEARN Studies possible. The complete A4 Study Team list is available on: a4study.org/a4-study-team. 
Conflict of Interest: R. Amariglio has nothing to disclose. J. Grill has nothing to disclose. D. Rentz has nothing to disclose. G Marshall reports personal fees and institutional support from Eisai Inc., institutional support from Eli Lilly and Company, Janssen Alzheimer Immunotherapy, Novartis, and Genentech, and personal fees from Grifols Shared Services North America, Inc, Pfizer outside the submitted work. R. Buckley has nothing to disclose. R. Yaari reports personal fees from Eli Lilly and Company during the conduct of the study. R. Raman reports grants from NIA, grants from Eli Lilly during the conduct of the study, grants from Janssen and grants from Eisai, outside the submitted work. CK. Sun reports grants from NIA and grants from Eli Lilly and Company during the conduct of the study. J. Sims he is an employee and stock holder of Eli Lilly and Company outside the submitted work. M. Donohue reports grants from NIH, grants and personal fees from Eli Lilly and Company during the conduct of the study, personal fees from Roche, personal fees from Biogen, personal fees from Neurotrack, other from Janssen, personal fees from Vivid Genomics outside the submitted work. P. Aisen reports grants from Janssen, grants from NIA, grants from FNIH, grants from Alzheimer's Association, grants from Eisai, personal fees from Merck, personal fees from Biogen, personal fees from Roche, personal fees from Lundbeck, personal fees from Proclara, personal fees from Immunobrain Checkpoint outside the submitted work. K. Holdrige reports she is an employee and minor stockholder of Eli Lilly and Company. S Sikkes reports grants from Zon-MW OffRoad, grants from EU-JPND, institutional support from Lundbeck, Boehringer, and Toyama outside the submitted work. Dr. Gatchel reports grants from Alzheimer's Association, grants from NIH/NIA, personal fees from Huron, grants from Merck, outside the submitted work.

Ethical Standards: Study protocols were approved by the Partners Institutional Review Board, and all participants provided written informed consent before undergoing any study procedures."

Open Access: This article is distributed under the terms of the Creative Commons Attribution 4.0 International License (http:// creativecommons.org/ licenses/by/4.0/), which permits use, duplication, adaptation, distribution and reproduction in any medium or format, as long as you give appropriate credit to the original author(s) and the source, provide a link to the Creative Commons license and indicate if changes were made.

\section{References}

1. Jack C, Bennett D, Blennow K, et al. 2018 NIA-AA Research Framework: Toward a biological definition of Alzheimer's disese. Alzheimers Dement 2018; 14: 535-562.

2. Administration USFD: Alzheimer's Disease: Developing Drugs for Treatment Guidance for Industry 2018.
3. Amariglio RE, Donohue MC, Marshall GA, et al. Tracking early decline in cognitive function in older individuals at risk for Alzheimer disease dementia: the Alzheimer's Disease Cooperative Study Cognitive Function Instrument. JAMA Neurol 2015; 72:446-454.

4. Gifford KA, Liu D, Carmona $\mathrm{H}$, et al. Inclusion of an informant yields strong associations between cognitive complaint and longitudinal cognitive outcomes in non-demented elders. J Alzheimers Dis 2015; 43:121-132.

5. Nuño, MM, Gillen D, Grill J. Study partner types and prediction of cognitie performance: implications to preclinical Alzheimer's trials. Alzheimer's Research \& Therapy 2019; 11:92.

6. Li C, Neugroschl J, Luo X, et al. The utility of the Cognitive Function Instrument (CFI) to detect cognitive decline in non-demented older adults. J Alzheimers Dis 2019; 60: 427-437.

7. Zwan MD, Villemagne VL, Dore V, et al. Subjective Memory Complaints in APOEvarepsilon4 Carriers are Associated with High Amyloid-beta Burden. J Alzheimers Dis 2016; 49:1115-1122.

8. Buckley, R, Ellis, KA, Ames, D, et al. Phenomenological characterization of memory complaints in preclinical and prodromal Alzheimer's disease. Neuropsychology 2015; 29:571-81.

9. La Joie R, Perrotin A, Egret S, et al. Qualitative and quantitative assessment of self-reported cognitive difficulties in nondemented elders: Association with medical help seeking, cognitive deficits, and beta-amyloid imaging. Alzheimers Dement (Amst) 2016; 5:23-34

10. Sperling RA, Donohue MC, Raman R, et al. Association of Factors With Elevated Amyloid Burden in Clinically Normal Older Individuals. JAMA Neurol 2020; e pub.

11. Walsh SP, Raman R, Jones KB, et al. ADCS Prevention Instrument Project: the Mail-In Cognitive Function Screening Instrument (MCFSI). Alzheimer Dis Assoc Disord 2006; 20:S170-17811.

12. Morris JC. The Clinical Dementia Rating (CDR): current version and scoring rules. Neurology 1993; 43:2412-2414.

13. Folstein MF, Folstein SE, McHugh PR. «Mini-mental state». A practical method for grading the cognitive state of patients for the clinician. J Psychiatr Res 1975; 12:189-198.

14. Wechsler D. WMS-R Wechsler Memory Scale Revised Manual, New York, The Psychological Corporation, Harcourt Brace Jovanovich, Inc, 1987

15. Benjamini, Y., \& Hochberg, Y. Controlling the false discovery rate: a practical and powerful approach to multiple testing. Journal of the Royal statistical society: series B (Methodological), 1995; 57: 289-300.

16. Snitz BE, Wang, T, Cloonan YK, et al. Risk of progression from subjective cognitive decline to mild cognitive impairment: The role of study setting. Alz \& Dement 2018; 14: 734-742.

17. Frank L, Lenderking WR, Howard K, et al. Patient self-report for evaluating mild cognitive impairment and prodromal Alzheimer's disease. Alzheimers Res Ther 2011; 3:35. 